Review

\title{
Magnetic Nanoparticles in Cancer Theranostics
}

\author{
Oliviero L. Gobbo ${ }^{1,2,4}{ }^{\bowtie}$, Kristine Sjaastad 3 , Marek W. Radomski1,4,5, Yuri Volkov ${ }^{3,6}$ and Adriele \\ Prina-Mello $3,6 \bowtie$ \\ 1. School of Pharmacy and Pharmaceutical Sciences, Trinity College Dublin, Ireland \\ 2. Trinity College Institute of Neuroscience, Trinity College Dublin, Ireland \\ 3. Department of Clinical Medicine Trinity Centre for Health Science, St. James's Hospital, Dublin, Ireland \\ 4. Trinity Biomedical Sciences Institute, Dublin, Ireland \\ 5. Silesian Medical University and Kardio-Med Silesia, Zabrze, Poland \\ 6. Centre for Research on Adaptive Nanostructures and Nanodevices (CRANN), Trinity College Dublin, Ireland
}

$\triangle$ Corresponding authors: Oliviero Gobbo, School of Biochemistry and Immunology, Trinity Biomedical Sciences Institute, Trinity College Dublin, 152-160 Pearse Street, Dublin 2, Ireland. Tel: +353 1896 3527; Fax: +353 1896 2783. Email: ogobbo@tcd.ie or Adriele Prina-Mello, Institute of Molecular Medicine, Trinity Centre for Health Sciences, St. James's street, Dublin 8, Ireland. Tel: +353 18963259 / 3087; Fax: +353 1896 3037. Email: prinamea@tcd.ie

C 2015 Ivyspring International Publisher. Reproduction is permitted for personal, noncommercial use, provided that the article is in whole, unmodified, and properly cited. See http://ivyspring.com/terms for terms and conditions.

Received: 2015.01.09; Accepted: 2015.07.15; Published: 2015.09.01

\begin{abstract}
In a report from 2008, The International Agency for Research on Cancer predicted a tripled cancer incidence from 1975, projecting a possible 13-17 million cancer deaths worldwide by 2030. While new treatments are evolving and reaching approval for different cancer types, the main prevention of cancer mortality is through early diagnosis, detection and treatment of malignant cell growth. The last decades have seen a development of new imaging techniques now in widespread clinical use. The development of nano-imaging through fluorescent imaging and magnetic resonance imaging (MRI) has the potential to detect and diagnose cancer at an earlier stage than with current imaging methods. The characteristic properties of nanoparticles result in their theranostic potential allowing for simultaneous detection of and treatment of the disease. This review provides state of the art of the nanotechnological applications for cancer therapy. Furthermore, it advances a novel concept of personalized nanomedical theranostic therapy using iron oxide magnetic nanoparticles in conjunction with MRI imaging. Regulatory and industrial perspectives are also included to outline future perspectives in nanotechnological cancer research.
\end{abstract}

Key words: Cancer, Magnetic nanoparticles, Drug delivery, Thermal therapy, Theranostic Nanomedicine

\section{Introduction}

In 1960, Richard Feynman gave a visionary talk about "smallness" and how miniaturization would lead to new technical applications.[1] He was already talking about nanotechnology and even pre-figured "nanomedicine" when he quoted one of his colleagues "it would be interesting in surgery if you could swallow the surgeon".[1] He was likely thinking about a "mini-robot" surgeon, however the idea of having a doctor inside a body is a good metaphor for the use of nanoparticles in medicine for diagnostic evaluation and/or treatment. Indeed, the list of the applications of nanomaterials in biology or medicine is immense.

In terms of biological imaging and diagnostics, the use of semiconductor nanocrystals (also called 'quantum dots') has resulted in the generation of bright, sensitive, extremely photo-stable and biocompatible semiconductor fluorophores, overcoming many of the limitations previously encountered by traditional organic dyes.[2, 3] For example, in Magnetic Resonance Imaging (MRI), it is possible to use iron oxide nanoparticles to detect liver metastases, metastatic lymph nodes, inflammatory and degenerative diseases [4] at an early stage when other contrast agents are unable to visualise them.[5] In multiple sclerosis (MS), ultrasmall particles of iron oxide (USPIO) detection preceded Gd-DTPA (gadolinium-diethylenetriamine penta-acetic acid) detection (preeminent MRI contrast agent) by 1 month.[6] Moreover, Vellinga and colleagues [6]showed that 
USPIO- and Gd-DTPA-enhancement disclose different pathological processes in MS. There are many other alloy/metallic nanoparticles that have potential biomedical applications, such as, manganese, nickel-gold alloy, which can be also coated with biocompatible materials similarly to iron oxide nanoparticles, the latter being the main focus of this manuscript.[7, 8]

In terms of therapeutics, there is a potentially large role for nanoparticles in tissue bone engineering. $[9,10]$ Nanoparticles can be also engineered for selective drug and gene delivery to targeted organs or tissues, minimizing exposure of healthy tissue to drugs or genes.[11-15] Furthermore, some nanomaterials are used for thermal therapy. Indeed, several classes of nanoparticles, including liposomes, magnetic and metallic, are currently in clinical trials for cancer thermal therapy.[16] A good example of this is the use of magnetic nanoparticles (MNPs), which can be designed to heat under a high frequency magnetic field to induce cancer cell death. $[17,18]$ Research progresses even further in the cancer fight by targeting cancer stem cells (CSCs). Indeed CSCs not only can play a major role in cancer initiation, progression and drug resistance, but chemotherapeutic drugs may increase the CSCs fraction in the tumour, allowing these cells to survive and evade to distant sites.[19] In this battle against CSCs, MNPs have shown encouraging results. For example, Sadhukha et al. discovered that the magnetic hyperthermia transduced by superparamagnetic iron oxide nanoparticles (SPION) in the alternating current magnetic field reduced or eliminated CSC population.[20] While the combination therapy of anti-ABCG2 monoclonal antibody and paclitaxel loaded iron oxide magnetic nanoparticles against cancer stem-like cell activity in multiple myeloma, led to significant reduction of tumour growth in a preclinical study.[21]

The aim of this review is firstly, to provide state of the art of nanomaterial application for cancer therapy, and secondly to advance a novel concept of personalized nanomedical theranostic therapy using multilayer magnetic nanoparticles in conjunction with MRI imaging. Indeed, magnetic nanoparticles (MNPs) constitute an important class of nanomaterials widely studied for their potential use in biomedicine fields (e.g. imaging, cell labeling, hyperthermia and drug delivery).[22] They can be classified as metal, alloys or oxides, and commonly consist of magnetic elements such as iron, nickel and cobalt and their chemical compounds. Iron-based NPs are the most studied, since iron is believed to be more biocompatible. Most of their applications are a consequence of their magnetic properties, which greatly differ from those of the bulk material.[22] The regulation and the industrial perspectives are also dealt with in the review, to de- lineate future perspectives of cancer nanomedicine.

\section{Multilayer concept}

The idea behind the multilayer concept is that a unique medical platform could have various properties from the detection to the cure of diseases. For example, the magnetic nanoparticle (MNP) surface can be engineered to achieve layering structure, to produce multi-functionalized nanoparticles for the prevention, diagnosis and treatment of diseases. Layering of iron oxide nanoparticles will generate functional SPIONs formed by three main components: (1) an iron oxide core functioning as MRI contrast agent, (2) a biocompatible coating and (3) a therapeutic coating targeted with a pharmacogenomics biomarker (Figure 1).[23-25]

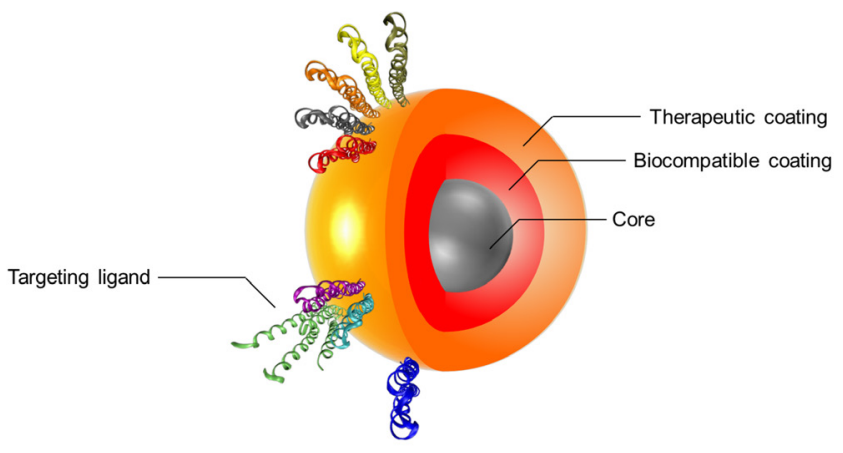

Figure 1. Schematic of a multilayered SPION with personalized medicine application. The figure depicts the three main components formed by "layering" an iron oxide nanoparticle: (1) engineered Nano-Particle (ENP) core, (2) biocompatible coating and (3) therapeutic coating/targeting ligand. Reproduced with permission from Handbook of Clinical Nanomedicine: Nanoparticles, Imaging, Therapy and Clinical Applications (Movia D, Poland C, Tran L, Volkov Y, Prina-Mello A. Multilayered Nanoparticles for Personalized Medicine: Translation into Clinical Markets). Copyright Pan Stanford Publishing Pte. Ltd. [25]

The careful preparation and consideration around each of the three components can allow for identification of the most suitable solution based on the expected performance of the multilayer nanoparticle. This will enable targeted accumulation in the site of interest (targeting component) allowing the diagnosis of diseases and the evaluation of the treatment efficacy (imaging/diagnosis component) while tracking the drug delivery and release to the targeted tissue/cell (personalized therapy component).

For instance, the use of SPION layered with active gene/drug compound as therapeutics carriers could improve the drug/gene delivery into the targeted tissues when compared to their simple gene or drug administration. This is intrinsically linked to the different delivery mechanism which in the case of SPION is avoiding the accumulation of drugs in healthy sites, which is commonly the cause of adverse side effects.[26] Within the multilayered concept, the therapeutics could be engineered into the SPION final formulation to protect against i) the body's defence 
system and ii) a premature degradation by a biocompatible coating.[26] To support this concept magnetite, as one of the most physiologically inert iron-based material is the most used compound for SPION particle synthesis.[26, 27] The synthesis of iron oxide nanoparticles has been intensively developed in the past years because of their technological interest for biomedical applications.[26-28] Numerous methods have been reported and explored to produce uniform nanoparticles with well-defined sizes, shapes, and other physical properties, using e.g. co-precipitation or thermal composition. [26, 27, 29] When exploited for industrial applications the multi-layered SPION has to comply with easy, clean and cost effective synthetic processes, and therefore in many cases ruling out methods in non-aqueous media.[30] Following the multilayer concept development process for biomedical application the most advantageous characteristic of iron oxide nanoparticles is the possibility to visualize them in the body using magnetic resonance imaging (MRI). At the basis of the multilayer concept the fine-tuning of the magnetic properties of the SPION can lead to change the relaxation rate of water protons in the immediate surroundings, exhibiting a change of contrast. [31, 32] This property has been successfully applied in cancer diagnostics as described below.

For clinical applications, it is of paramount importance that nanoparticles are also biocompatible. In fact, nanoparticle coatings is one of the most utilised strategy to prevent and or reduce any toxicity the SPION core might present and, therefore, reducing undesired toxic side effects. [33] The coatings are so important that they could modify the cell interaction process, pharmacokinetics and biodistribution of the same iron oxide nanoparticle. [29, 34] For instance, Ruiz and co-workers showed that the residence time was doubled for iron oxide nanoparticles coated with polyethylene glycol (PEG) as compared to the same bare nanoparticles. This resulted in reduced particle accumulation in liver and spleen.[35] Therefore, the role of the biocompatibility layer during the SPION development is to i) protect the therapeutic and targeting agents against the body's defence system, ii) improve the delivery efficiency of the cancer therapy and iii) avoid unwanted accumulation in secondary organs.

Overall, the use of nanoparticles as carriers for therapeutic and targeting molecules has been explored over the past 2 decades aiming at improving the therapeutic effect of the drugs and reducing their side effects. The engineering of SPION as multifunctional systems, loaded with various drugs and targeting molecules to direct them to cancer cells has been adopted for controlled drug release. [36] An- ti-cancer drugs such as Doxorubicin (used to treat e.g. breast, stomach and bladder cancer) or Gemcitabine (used to treat e.g. pancreatic, non small cell lung cancer and bladder cancer) can be attached to iron oxide nanoparticles using tailored linkers. These ones when internalized into the cell, could be sensitive to certain intracellular triggering stimulus such as $\mathrm{pH}$, enzymes or external stimuli such as temperature and release drugs from magnetic nanoparticles in a controlled manner.[36] DNA and RNA could also be encapsulated onto SPION for cancer gene therapy.[37] In the context of a therapeutic action, antibodies have interesting properties, since they can mediate the destruction of cancer cells by the body's immune system and be used as targeting agents. Indeed, they are specific and would be directed at their tumour targets (antigens) without affecting or with only minimum effect on healthy tissues.[38] They are other targeting molecules such the Nucant pseudopeptide (N6L), which binds nucleolin, a protein overexpressed in the membrane of cancer cells. N6L acts also as an anticancer drug, by entering the cell nucleus to induce apoptosis.[39]

When compared to conventional pharmaceutical products, nanometric drug delivery systems can often lead to higher drug bioavailability with lower dose (thus decreasing the adverse side effects). In addition, targeting moieties decorating the Engineered Nano-Particle surface can direct efficiently the clinical treatment to the affected tissue/cell with optimal selectivity.[40]

Finally, the adoption of the multilayer concept can be linked to improved safe-by-design and green-by-design approaches with the view of reducing the regulatory process for product approval.[25]

\section{Cancer Diagnostics}

In 2008, the International Agency for Research on Cancer released its report on world cancer and noted that the global number of cancer cases doubled between 1975 and 2000 and it is expected to triple by 2030, leading to a projection of between 13 to 17 million cancer deaths.[41] Early detection of cancer greatly increases the chances for successful treatment. Screening across a healthy population may help to identify individuals who have the disease, but who do not yet have symptoms (such as in e.g. breast or cervical cancer). However, some cancers (such as pancreatic cancer) are difficult to detect with screening and, therefore, an early diagnosis is the only chance to detect tumour cell growth.

Nanotechnology is likely to play a major role in cancer diagnosis by allowing the visualisation of the cancer cell at an early stage. A few years ago, Ralph Weissleder described the advancement of molecular 
imaging in cancer[42], and predicted that medical imaging technologies will play a central role in clinical oncology. Molecular imaging will allow clinicians to see not only where a tumour is located in the body, but also to visualize the expression and activity of specific molecules that influence tumour behaviour and/or response to therapy.[42] Indeed, modern clinical cancer diagnosis requires precise information, such as the position and the size of the tumour, and also if there are indications of metastases. However, progress in cancer imaging is relatively slow because of a poor pharmacokinetics profile or a high cost of the clinical development of molecular imaging agents.[42] It seems, therefore, that successful imaging agents must show improved pharmacokinetic profile, display low toxicity and be manufactured by cost-effective manufacturing techniques to be of relevance to clinical practice.

In the last few decades, cancer imaging research has seen a growth in the number of different imaging technologies, providing anatomic and physiological information, which are now in widespread clinical (e.g. positron emission tomography (PET) and single-photon emission computed tomography (SPECT)) and preclinical (e.g. fluorescence reflectance imaging (FRI)) use.[43] All of these techniques demand that the targeted molecules or the cells become visible. The three main strategies use genetic reporters (e.g., photoproteins or PET and MR detectable reporter genes) or radiotracer, fluorochrome, or magnetically tagged affinity molecules (e.g., labelled antibodies or small molecules) or biorthogonal reporter strategies.[43] In this context, nanotechnology could play a major role in delivering new imaging probes. Indeed, nanoimaging has the potential to detect and diagnose cancer at an earlier stage than with current imaging methods.[12]

Currently, the two main applications in nanoimaging are fluorescence imaging and Magnetic Resonance Imaging (MRI). In fluorescence imaging, quantum dots (QD) are used to visualise target tissue or to study the lymphatic system. These colloidal semiconductor nanocrystals can be administered in an animal and excited in vivo with a long wavelength. They are then detected by their resulting fluorescence using a sensitive charge-captured device camera.[12] While initial studies of the in vivo toxicity of QDs suggested that QDs are safe, a recent publication found clear signs of cytotoxicity for cadmium based-QDs.[44]

MRI is a non-invasive, tomographic imaging modality and is based on the manipulation of the inherent nuclear magnetic moment of endogenous nuclei. Images are obtained by exposing nuclei to a static magnetic field and, within that static field, perturbing a steady-state equilibrium with time and space varying the magnetic fields. After perturbation, all nuclei relax by two unique and co-dependent relaxation mechanisms: T1 (spin-lattice relaxation) and T2 (spin-spin relaxation).[43] In the presence of MRI contrast agents, the relaxation times of protons nearby tissues change and create a modification in the relaxation time reading, which can be efficiently detected by MRI. There are numerous contrast agents or new applications for existing contrast agents.[45] However the 3 mean magnetic labels used in vivo MRI are gadolinium (Gd)-based contrast agents, hyperpolarized molecules and iron oxide magnetic nanoparticles. Gd complexes, despite their nephrotoxicity [46] are frequently used as a MRI contrast agent to enhance images in medical magnetic resonance imaging procedures.[46] Hyperpolarized molecules have a more recent history in MRI. The main molecules used are hyperpolarized gases ( ${ }^{3} \mathrm{He}$ and $\left.{ }^{129} \mathrm{Xe}\right)$, which allow for MR images of lungs for indirect visualization of pulmonary diseases to be obtained.[47, 48] Other hyperpolarized molecules such as ${ }^{13} \mathrm{C}$ are offering the possibility of obtaining information about molecular behaviour in vivo.[47] Whatever the molecule used, signal enhancement from the hyperpolarized species has a transitory nature, which makes these techniques a little more sophisticated to use in routine MR imaging than other magnetic labels. In this context, NPs can be successfully exploited to improve medical imaging for diagnostic purposes.

Nanoparticles, such as gold NPs and quantum dots, can enable the detection of tens to hundreds of cancer biomarkers in blood assays or on cancer tissue biopsies.[15] However, as it stands, these NPs are not currently suitable for human in vivo diagnostics imaging. In contrast, magnetic nanoparticles (MNPs) are already used in clinical diagnosis as a magnetic resonance imaging (MRI) contrast agent.[49] Two distinct classes of magnetic NPs are currently used for clinical imaging: ferromagnetic iron oxide particles and ultrasmall superparamagnetic iron oxide particles.[4] They work by shortening the spin-lattice relaxation time $\mathrm{T} 1$ and spin-spin relaxation time $\mathrm{T} 2$ resulting in the formation of a sharper and brighter image.[50] Due to their superparamagnetic properties, these NPs can change the spin-spin relaxation time of the neighbouring water molecules and then monitor the expression of genes, detect tumours, and other diseases.[11] They can be actively targeted or passively targeted to differentiate between the normal and the diseased tissue.[11] Magnetic NPs are, therefore, the most suitable NPs in terms of rapid availability for in vivo human diagnostics and imaging of cancer. They also show the immense advantage of selectively delivering a drug to the cancer cells.[51] We will provide 
more detail on their proprieties in the next section. Although MNPs are not the only nanoparticles combining drug delivery and imaging[11], they are the most advanced in the process of research and development as therapeutic agents as well as MRI contrast labels in humans.[11, 15] For example, Hayashi et al. [52] have shown in a very elegant set of experiments the advantages of SPION i.v. administrated for cancer theranostics by combining MRI as diagnostic imaging modality and magnetic hyperthermia treatment. Previously Zhao et al. [53] demonstrated the benefits of intratumour injection of SPION for hyperthermia effect, showing MR images of the SPION-labelled tumour.

\section{Cancer Therapeutics}

Surgery, radiotherapy and chemotherapy are the key components of cancer treatment. Chemotherapy uses cytotoxic agents to target malignant tumours in organs or tissues. Unfortunately, chemotherapy is expensive and in addition to cancer affects normal cells leading to numerous side effects. By using nanoimaging and nanodrug delivery systems cancer cells can be selectively targeted thus reducing undesired systemic drug toxicity. Nanoparticle-based drug delivery systems, especially liposomes [54, 55], have already been approved by the US Food and Drug Administration (FDA) for the treatment of specific cancers[56] and continue to be developed in pre-clinical research.[57] Here, we will provide more information on the use of MNPs in cancer therapeutics.

The most widely used MNPs are magnetite $\mathrm{Fe}_{3} \mathrm{O}_{4}$ and maghemite $\gamma-\mathrm{Fe}_{2} \mathrm{O}_{3}$. Pure metals such as $\mathrm{Fe}$, $\mathrm{Ni}$ and $\mathrm{Co}$, ferrites of the form $\mathrm{MeO} @ \mathrm{Fe}_{2} \mathrm{O}_{3}(\mathrm{Me}=\mathrm{Mg}$, $\mathrm{Zn}, \mathrm{Mn}, \mathrm{Ni}, \mathrm{Co}$, etc) may be also used to prepare MNPs.

\section{Drug delivery}

Magnetic nanoparticles appear to be very appropriate for drug delivery. Indeed, they can be synthetized in different sizes and can be functionalized (surface-coated) in order to carry various molecules. A number of issues need to be considered when using MNPs as carriers including colloidal stability and biocompatibility.[58] Furlani [58] has recently described the properties of nanomaterials in the physiological environment and their specific properties used during the elaboration of these nanoparticle-based drug-delivery systems. Indeed, MNPs need to be coated with surfactants or polymers (e.g. dextran, polyethylene glycol) to stabilise them and attach functional groups to their surfaces. The functionalisation is used to bind the appropriate molecules, such as anti-cancer drugs or antibodies, to the nanoparticles.[55] Surfactants and polymers increase also the biocompatibility of the nanoparticles. Indeed, without this shield MNPs could not resist opsonisation (i.e. the process by which an exogenous molecule is tagged for destruction by phagocytosis) when introduced in vivo.[55] Another advantage of these nanoparticles is that they are nontoxic and well tolerated in vivo, independently of the administration routes.[58-61]

Nanoparticles are already widely used in drug delivery, offering to transport various agents such as antimicrobial molecules, genes, proteins and anti-cancer drugs. Many chemotherapeutic drugs and siRNA treatments have already been loaded in different nanoparticles and have demonstrated a great efficacy against different types of cancers [62-64], some are even in phase I of drug development studies. While cancer drug delivery via MNPs is seen in its infancy (to date, only a few reports with in vivo results have been published), it has great potential due to the numerous advantages of MNPs. For example, recently, Maeng et al. [65] have shown promising results MNPs loaded with doxorubicin (a potent anti-cancer agent) against liver cancer in rat and rabbit cancer models.

Gene therapy represents an alternative to anti-cancer drug treatment for cancer. Indeed, this therapy could target directly genes and regulate the altered gene expression, which is involved in carcinogenesis. However, in vivo, the half-lives of DNA or RNA are very short which makes gene therapy delivery very challenging. Nanoparticles when used for drug delivery should, therefore, have a dual function, the first is to protect the therapeutic agent from degradation and the second is to deliver it to cancer cells. Magnetic nanoparticles technology has one more advantage; it attracts NPs near the tumour and increases gene transfer into cells.[66] This technique is called magnetofection (Figure 2) and uses an external magnetic field (magnet) to concentrate and retain MNPs in a specific area.[67] However, this approach is not applicable for non-accessible tumours.[67]

\section{Thermal therapy}

For the treatment of cancer, thermal therapies have numerous advantages. The technique is focal and repeatable with a minimally invasive application. There are 2 main types of thermal therapy: cool- or heat-based techniques. Cryosurgery is based on the application of extreme cold to destroy tumours e.g., in liver, prostate lung cancers. In recent years, the use of nanoparticles has led to a new technique called nanocryosurgery [68] which was proposed to improve freezing efficiency of the conventional cryosurgery. 


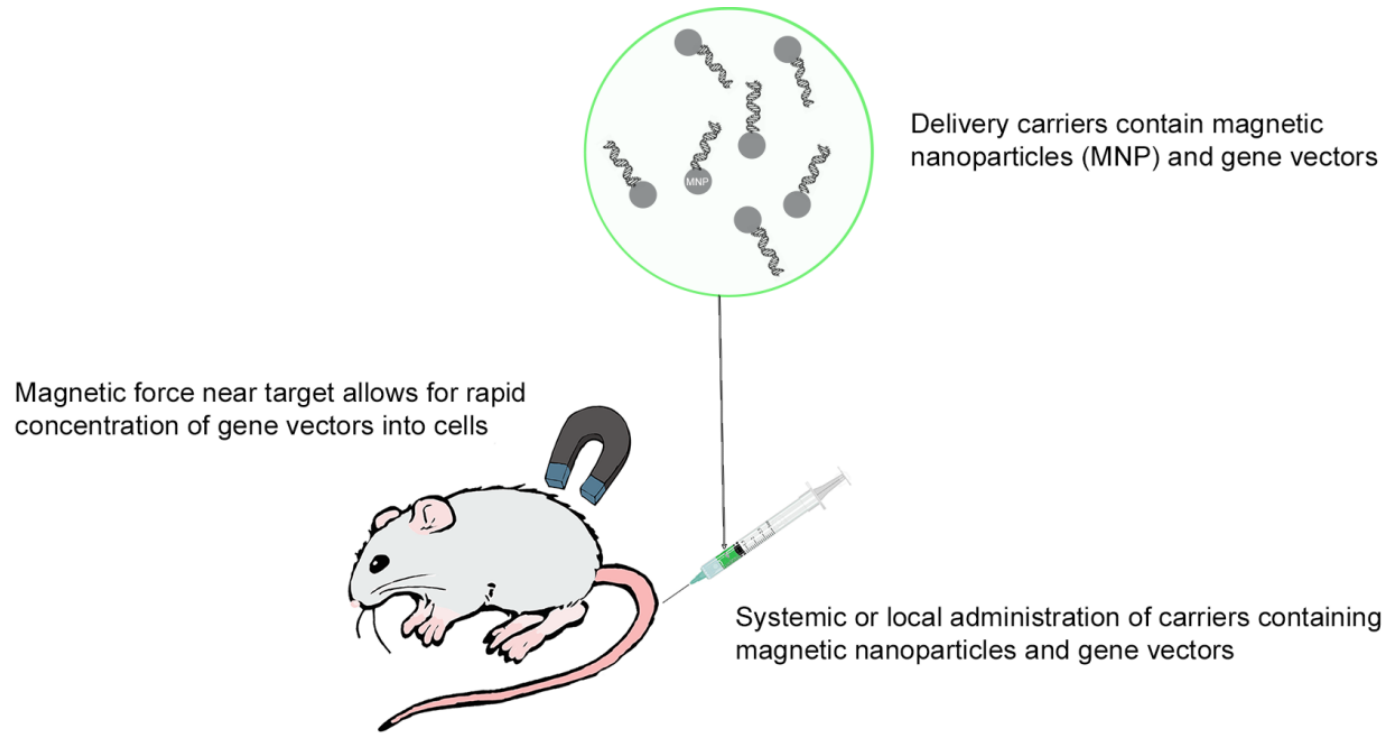

Figure 2. Overview of in vivo magnetofection.

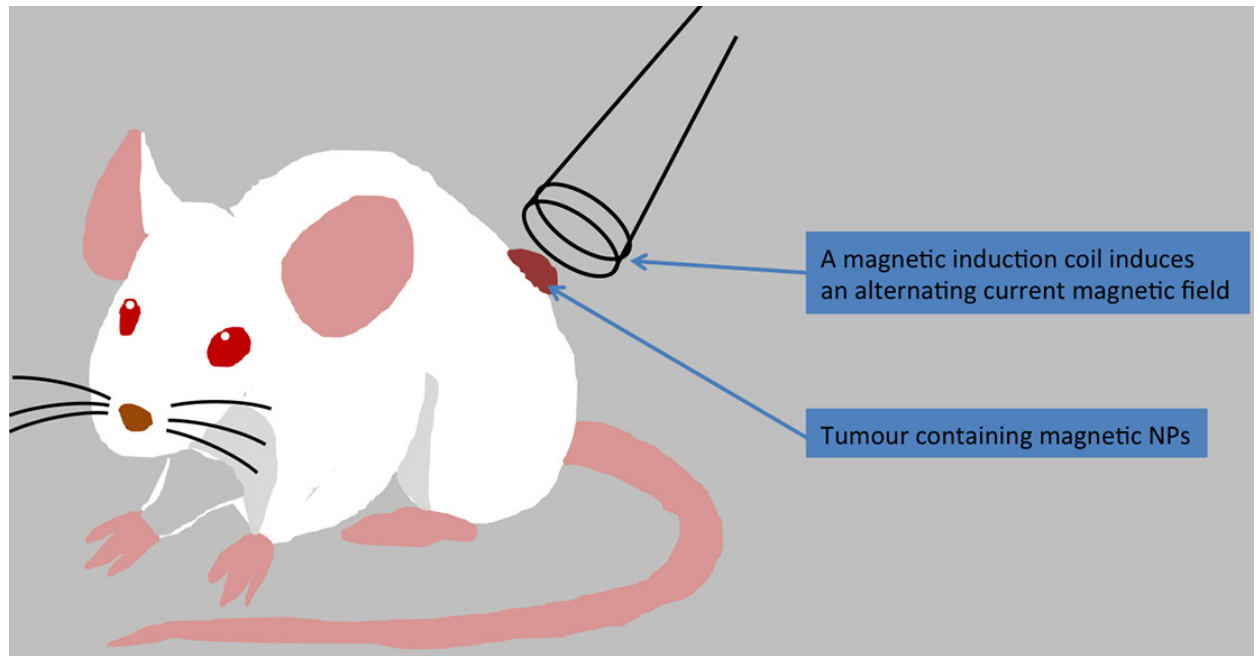

Figure 3. In vivo magnetic hyperthermia treatment in mouse. MNPs could be injected intravenously or directly into the tumour of a mouse and an applied alternating current magnetic field increases temperature in the tumour tissue. The temperature reached in the tumour centre depends on the nanoparticle concentration.

While the use of MNPs in cryosurgery is at its infancy, in hyperthermic treatments MNPs have been investigated for decades.

For hyperthermic treatment, different techniques are available, such as lasers [69, 70], high intensity focused ultrasound [71, 72], radiofrequency currents $[73,74]$ or alternating magnetic field. [75-77]

Nanoparticles have been also investigated to deliver thermal energy to tumours. [78] The different techniques use the properties of NPs inherent to their size and composition such as optical and magnetic characteristics, thermal or electrical conductivity. For example, photothermal therapy uses laser light to heat NPs to selectively kill cells which incorporated these NPs. [79] More recently, graphene-based nanosystems have been shown to improve the stability, bioavailability, and photodynamic efficiency of organic pho- tosensitizer molecules. Owing to its intrinsic high optical absorption in the near-infrared region, functionalized graphene oxide can combine both photodynamic and photothermal hyperthermia for optimum therapeutic efficiency.[80] In a recent review article $\mathrm{Li}$ and co-workers [80] have reported the current progress in the photodynamic therapy via nanotechnology.

The use of an external magnetic field is another technique to treat cancer with magnetic NPs. Indeed, minimally invasive magnetic heating therapy uses superparamagnetic iron oxide MNPs to generate heat (with an external alternating magnetic field) to specific tumour areas (Figure 3). So far, different cancer types e.g. brain, breast, prostate, and liver cancers have been treated using this technique. A great advantage of this approach is that MNPs can be injected 
directly into the tumour before heating treatment and MNPs seem to retain almost completely in the tumour allowing for repeated therapeutic sessions.[76] MNPs can be also administered to the bloodstream, but for such treatment MNPs would need to be targeted to the tumour before the heating therapy. In the following section, we will describe the different strategies, which can be used to target and treat tumours including glioblastoma.

\section{Direct injection of MNP into the tumour}

Although most commercially available products using nanoparticles are found as contrast agents, recently milestones have been reached regarding anticancer therapy. The German company MagForce released their company presentation at the healthcare conference in May 2013, announcing the European Medicines Agency (EMA) approval of a new product, NanoTherm as a treatment of primary or recurrent glioblastoma multiforme, a lethal brain tumour with limited treatment options.[81] The new treatment involves the use of an aqueous dispersion of iron oxide nanoparticles, which is injected into the tumour. Once the particles are safely transferred, an alternating magnetic field applicator is used to alternate and adjust the magnetic field strength, thus creating heat and delivering localised treatment. The particles are coated with aminosilane, and a high concentration of iron with magnetic core causes less amounts of fluid to be injected for appropriate temperature generation to be achieved.[25, 82] A number of contributions have been published showing pre-clinical and clinical data obtained using this platform.

In rats aminosilicane coated particles when injected intra-tumourally to the rat glioblastoma prolonged survival 4.5 fold. These particles remained in the tumour after injection, thus enabling serial thermotherapy without the need for repeated injections. The anticancer effect was seen from necrotic cancerous tissue surrounding the particles, as well as a decreased proliferation rate.[83]

Maier Hauff et al.[82] combined MNP with external beam radiotherapy in 66 patients with recurrent glioblastoma multiforme. The trial showed that the MNP treatment was safe and effective, with longer overall survival reported in results.[82] NanoTherm has currently been released in 27 European countries, and the treatment is available to limited number of patients on a case-by-case basis.[81]

Following successful achievements of NanoTherm for glioblastoma multiforme, a new clinical trial is currently on going to document post-marketing results of its treatment effect. Furthermore, research using similar treatment has been started for other solid tumours with limited treatment options. A Phase I clinical trial establishing toxicological effects of intratumoural iron oxide nanoparticles for hyper thermic treatment of patients with locally recurrent prostate cancer has been completed, showing promising results.[84]

\section{Targeted drug delivery}

\section{Passive targeting}

Passive targeting may be considered very important since the free accumulation of MNPs in the tumour area could increase the MRI detection and facilitate the diagnosis of cancer. Indeed, the tumours develop their own blood supply from the existing vascular system. Cancer-induced angiogenesis produces abnormal vessels which present fissures and through this state of leaky vasculature, NPs may accumulate in the tumours.[56] Nevertheless, passive targeting depends also on different physicochemical properties of the NPs such as the particle size, the surface charge and hydrophobicity.[85] Indeed., small NPs $(<20 \mathrm{~nm})$ are excreted by kidneys while medium sized NPs (30-150 nm) have been found in the bone marrow, heart, kidney and stomach. In contrast, large NPs tend to accumulate in the liver and spleen. Moreover, solid tumours show the enhancement permeability and retention (EPR) effect for NPs of an appropriate size. The EPR effect is due to the defective vasculature and deficient lymphatic drainage system in solid tumours causing increased pore size in the endothelial wall.[85] In consequence, large NPs do not readily penetrate to the tumour while small NPs can easily enter and leave the tumour. It is suggested that NPs ranging from approximately 10 to $100 \mathrm{~nm}$, preferentially accumulate in the tumours as opposed to normal tissues.[85] Concerning the other properties of NPs, it seems that hydrophobic and positively charged molecules have short circulation times due to opsonisation, while hydrophilic and negatively charged NPs have long circulation times.[86] In summary, although the leaky vasculature is an enormous advantage for targeting tumours, passive targeting may not allow for sufficient amounts of NPs to accumulate in the tumour area for successful molecular MRI and treatment.

\section{Active targeting}

A promising strategy to reach tumours is the development of active targeting of cancer cells by magnetic drug treatment or through interaction mediated by ligands such as antibodies folate, lectins and peptides as presented with NPs. In magnetic drug treatments, the drug or other active molecules is conjugated on a surface of a magnetic nanoparticle or could be encapsulated with a magnetic molecule into a nanoparticle platform such as liposomes, micelles or 
dendrimers. $[55,85]$ Magnetic drug targeting has been studied in tumours [55] and various diseases. An external magnetic field applied on surface tumours, should attract and maintain MNPs loaded with active molecules into the area of interest. Animal experiments $[87,88]$ and clinical trials have produced very consistent results in cancer treatments.[55, 89] An external magnetic force can also improve delivery of a magnetic targeted aerosol to specific lung regions other than the airways or lung periphery. The inhalation of a magnetic aerosol can be used not only for the treatment of lung cancer but also in other non-malignant pathologies such as chronic obstructive pulmonary disease, cystic fibrosis and respiratory infections.[90]

Another way to increase accumulation of anticancer drugs into cancer is to use NP platforms with biotargeting agents that bind specifically to tumour cells. The use of a magnetic NP platform will not only increase drug concentration in the tumour, but also reveal the tumour itself in MRI. This is a very important feature, especially at an early stage of the disease, when the cancer is not yet diagnosed, but there are grounds for suspecting a cancer. Various ligands are used to target cellular biomarkers expressed or over expressed by the malignant cells. Antibodies can be used as cancer active agents, but because of a lack of direct cytotoxic effects, they are commonly used to bind cancer targets with high affinity. Monoclonal antibodies can target various receptor proteins including endothelial growth factor receptors, and vascular endothelial growth factor receptors which are overexpressed or abnormally activated in tumour cells.[91] Peptide ligands with high affinity toward surface receptors of cells can also be used to target tumour cells that over express these receptors. For example, Integrins are distributed in a variety of tissues and endothelial cells and their expression is increased in cancer. In recent years, many efforts have been focused to develop integrin ligands to target integrin receptors in pathological states.[92] Peptide ligands can also be used for cancer diagnosis. For example, chlorotoxin, a peptide with a high selectivity for membrane-bound matrix metalloproteinase-2 (MMP-2), is over expressed in various cancers such as glioma, prostate and intestinal cancer.[93] Huang and colleagues [93] built a macromolecular platform carrying chlorotoxin and a MRI contrast agent $\mathrm{Gd}$, to target specific cancers. In nude mice bearing C6 glioma or liver tumour models, they showed that their MR imaging platform could be used to diagnose MMP-2-expressing tumours. One can, therefore, envisage an anticancer nanopaticle platform with a MRI contrast agent and a double action peptide ligand. Such ligand could selectively bind to cancer cell surface proteins and trigger off cell death. The best example is a synthetic ligand of cell surface nucleolin known as N6L.[94] Indeed, surface nucleolin expression is enhanced in various tumour cells (a selective target) and plays a role in the apoptotic process (antitumour activity).[94]

\section{Regulatory}

\section{Toxicity Regulation and environment}

Since the development of nanoparticles for medical applications, researchers have been in disagreement regarding the level of toxicity related to Iron Oxide Particles (IOP).

The accurate estimation of cytotoxicity by conventional methods is difficult, due to the complexity and variability within the individual parameters of each nanoparticle.[95] A standardised and comparative method of assessment has therefore been difficult to establish. The development of such method is a priority of the European Medical Agency who states that "specific guidance on quality, toxicology, clinical development and monitoring aspects may be developed in the future, once sufficient scientific experience has been gained for specifically identified sub-technologies within the field of nano medicines".[96]

In 2009, The International Regulators subgroup on nano medicines was launched comprising a global panel of experts. The European Union (EU) was represented by the EMA, USA by FDA, Japan with representatives from the Ministry of Health \& Labour and Welfare, and Canada through Health Canada representatives. Their main aim was to share their knowledge in order to appropriately move forward with safer products made available for clinical application.[97]

An essential component to toxicological regulation of nanoparticles is to establish the appropriate classification as a medicinal product or a medical device. As the regulation of these products varies, an inaccurate classification could potentially prevent certain products from achieving marked authorization. The different definitions are outlined in the table 1.

A normal drug/medicinal product is usually approved after an initial in vitro screening to rule out significant adverse events and to ensure sufficient biocompatibility of the product. If appropriate safety levels are achieved, in vivo screening is completed before FDA or EMA approval is sought.[96]

The French Agency for the Safety of Health Products released recommendations for pre-clinical toxicity testing of nanoparticle medicinal products in 2011. Firstly, emphasis was made on establishing suf- 
ficient knowledge of the pharmacokinetics of the nanoparticle, including its absorption, distribution, metabolism and excretion. Furthermore, safe pharmacology could be achieved through single and repeated dose toxicology studies, as well as case-by-case protocols attempting to recreate human exposure conditions as much as possible. The necessity of in vitro toxicity was emphasised, with attention drawn to effect on cytotoxicity, phagocytic capacity, and macrophage or complement system activation leading to generation of free radicals. Specific toxicology measurements to be established in pre-clinical testing are further outlined in the table 2.[100]

Table 1. Outlining the definitions of a medicinal product and a medical device as stated by the European Commission.[98, 99]

Definition of a drug or Medicinal product as stated by the European Commission.

"Any substance or combination of substance presented as having properties for treatment or prevention of disease in humans, or any substance or combination of substance used or administered to humans with the view to restore, correct or modify physiological function by exerting pharmacology, immunology or metabolic action or to make a medical diagnosis. "

Definition of a Medical Device as stated by the European Commission. "Any instrument, apparatus, appliance, software, material or any other article, used alone or in combination, including software intended by manufacturer to be used specifically for diagnosis or therapeutic purposes \& necessary for proper application intended by manufacturer, to be used in human beings with the purpose of:

diagnosis, prevention, monitoring, treatment or alleviation of disease, diagnosis, monitoring, treatment, alleviation of or compensation for an injury or handicap, investigation, replacement or modification of the anatomy or of a physiological process, control of conception, and which does not achieve it's principal intended action in or on the human body by pharmacological, immunological or metabolic means, but which may be assisted in it`s function by such means."

Table 2. Areas of specific toxicity established by the non-clinical testing guidelines. It is important to establish the effect each novel nanoparticle will have on these areas before proceeding to clinical testing of the product.

Areas of Potential Specific Toxicity Development from use of Nanoparti-

cles

1. Immunotoxicity

- Both the innate and adaptive immune system

- Generation of reactive oxygen species

- Cytokine release

- Hypersensitivity

- Immunosuppression

2. Formation of Agglomerates

3. Reproductive Toxicity

4. Genotoxicity

5. Carcinogenic potential

There is limited correlation between in vivo and in vitro nanoparticle toxicity, as in in vitro experiments the complexity of the body's homeostatic response cannot be fully assessed.[101]

On this matter, several reflection papers have been published from the European Medical Agency addressing the regulatory environment evolving around commercially available iron oxide nanoparticles. The reflection paper from 2011 on non-clinical studies for the application of iron oxide nanoparticles states that a normal medicinal product will achieve approval through the comparison of physicochemical features, as well as the plasma concentration of the active ingredients to the generic reference product, as these will not significantly differ. This approach cannot be followed regarding nanoparticles, as their variation in properties is likely to change their level of toxicity. They, therefore, claim that pre-clinical studies are necessary for the approval of each individual generic nanoparticle.[102]

It is evident that for using IOP for medicinal products, plasma, reticuloendothelial system (RES) and target tissue concentrations of both free iron and particles must be established and assessed. Additionally, pre-clinical studies must be completed determining the sensitivity of cell assays used as well as the degradation of the nanoparticle in comparison to other reference products.[102]

Another reflection paper published by the European Medicines Agency in 2013 includes additional focus on the coating of the particles. It is important to consider the effect coating of the particles will inflict on the safety and efficacy, as it affects the pharmacokinetics, biodistribution and intracellular fate of the particles. There are important characterisations that must be established during the manufacturing of the coated nanoparticles, in order for approval to be considered.[103]

Once pre-clinical testing has been completed appropriately as seen through the section above, further clinical evaluation is necessary in order for the nanoparticle to reach authorization status. The European Medicines Agency, through the Committee for medicinal products for human use, produced guidelines on clinical evaluation of all diagnostic agents in 2009. From these guidelines, it is clear that when introducing new diagnostic agents to the marked, it is not enough to simply achieve similar results as previously approved agents. The new agent must display a clinical benefit, further being demonstrated through technical and diagnostic performance, as well as a sufficient effect on diagnostic thinking. Additionally the patient management and clinical outcome can be of relevance. The Technical performance is measured by the quality of images in comparison to gold standard agents, as well as benefits related to the procedure of the agent. Diagnostic performance is established through statistical measurements, where improved sensitivity and specificity of a diagnosis is expected. Furthermore, the probability of correct diagnosis must be considered, seen in the effect on di- 
agnostic thinking. According to these guidelines, all appropriate aspects of a protocol must be completed in a stage III trial. Information of sensitivity and specificity should be established in phase I/II trials, and should be compared to the potential benefit of the new diagnostic agent. For ultimate approval to be granted, a risk/ benefit ratio will be completed. An appropriate technical and diagnostic performance of the new agent will be compared to the standard therapy available, where a positive effect must be established.[104]

\section{Role of theranostic nanomedicine in can- cer treatment}

Nanomedicine has applied nanotechnology in various medical fields such as imaging, diagnosis of or therapy in human diseases. Theranostics combines the last 2 fields, while theranostic nanomedicine produces "nanoparticle-based drugs" simultaneously capable of the diagnosis and treatment of a disease.

The goals of theranostic nanomedicine, therefore, are not only to improve the detection and to increase the efficacy of the treatment of cancers but also to limit the systemic toxicity associated with this treatment. It is, therefore, important that the therapeutic agents reach and can be concentrated on the target sites. Another important advantage of theranostic nanomedicine in the treatment of cancer is the potential for a rapid review of the outcome of a treatment in individual patient, in order to plan the next therapy or to decide to repeat the same therapeutic session (personalized medicine).

We feel that the use of magnetic NPs in conjunction with MRI imaging may advance the concept of personalized nanomedical theranostic treatment in cancer for an individual patient.[105] As MRI scanners are nowadays readily available in hospitals this seems to be the most appropriate technique for monitoring the effects of cancer nanomedicine therapies.[106] Moreover, magnetic NPs have an enormous advantage in this type of therapy, due to their paramagnetic propriety: they can be easily used as a contrast agent.[107] We described in this review three other advantages of MNPs. The first is the active targeting of these NPs: they can be concentrated at a defined area by a local application of an external magnetic field.[55, 87-90] The second is that MNPs can be used as heat-generating systems for hyperthermic treatments.[75, 76] Finally, they seem to be well-tolerated in vivo.[58-61] Therefore, MNPs combine already four important features as a therapeutic agent in oncology. As cancers often require multiple treatments (e.g. surgery + chemotherapy or thermotherapy + radiotherapy [82]) to successfully eliminate malignant tissues, combining MNPs with different anticancer agents such as doxorubicin $[90,108]$ or the synthetic ligand N6L [94] can provide a nanomaterial-based agent with a dual-effect (chemotherapy + thermotherapy) against various types of cancer. One can also load MNPs with more than one anticancer agent (e.g. doxorubicin or siRNA). It has been shown that multifunctional MNPs loaded with N6L have an important advantage in comparison to "classic" anti-tumour drugs since N6L binds to the cell surface proteins expressed in various malign cells.[94] With these multifunctional MNPs, it is possible to use two active targeting systems (magnetic field + targeting agent). The use of this double active targeting can, therefore, increase the concentration and the retention time in targeted tissues thus improving the efficacy of the anti-cancer properties of these MNPs.

The challenge now is to design the most appropriate MNPs for theranostic applications. The key features of an "ideal candidate" are indicated in Figure 4 .

\section{European investment on magnetic carri- ers for the medicinal industry}

From the extensive research carried out in the past decades it is clear that a substantial amount of time and financial investments have been made to explore the potentially revolutionizing medical applications iron oxide nanoparticles.

This unified European research on the development of new nano-carrier and medicinal products has been mainly supported by the European Commission under the past the seventh framework programme (FP7) and now Horizon2020 for research and development. The European Union has so far contributed with over 400 million euro funding 85 projects within nanosciences, nanotechnologies, materials and new production technologies through the FP7-NMP programme. Many of these projects are based on iron oxide nanoparticle development and MR imaging, as outlined in table 3.[109]

More than half of these projects are focused on cancer research. For example, NanoTher wishes to fight cancer by developing a functionalized nanoparticle with theranostic attributes. The theranostic potential is further investigated through Magnifyco, a project group wanting to develop nanostructures for treatment of ovarian cancer. These structures will contain magnetic properties, enabling them to function as MRI contrast agent, hyperthermic treatment and improving drug release. These structures will be encapsulated, and contain surface antibodies specific to ovarian cell lines. Nanomagdye uses iron oxide nanoparticles with submicron bubbles and dye for imaging within oncology. They wish to develop a new contrast agent for better sensitivity of lymph node 
imaging. Namdiatream is an inter-disciplinary research group comprising of 22 different partners. Their aim is to develop detection of biomarkers related to different cancer types for early diagnosis and treatment. SaveMe aims to fight cancer by creating nano core platforms to create theranostic potential. Multifun wishes to provide an earlier detection of breast and prostate cancer, by combining iron oxide nanoparticles with cancer stem cells. Finally, NanoFol aims at improving treatment options for inflamma- tory diseases by enabling siRNA delivery to activated macrophages.[110]

An additional 31 projects are funded through FP7-Health, contributing to an additional 150 million euro. Further nano medicine related projects are funded by sub programmes, such as PEOPLE, the European Research Council or Eurostars.[109]

From this it is clear that the European Commission has made significant efforts to develop safe commercially available products within nanotechnology.[110]

\section{THERAGNOSIS: MRI detection + Multimodal Therapeutic Approach}

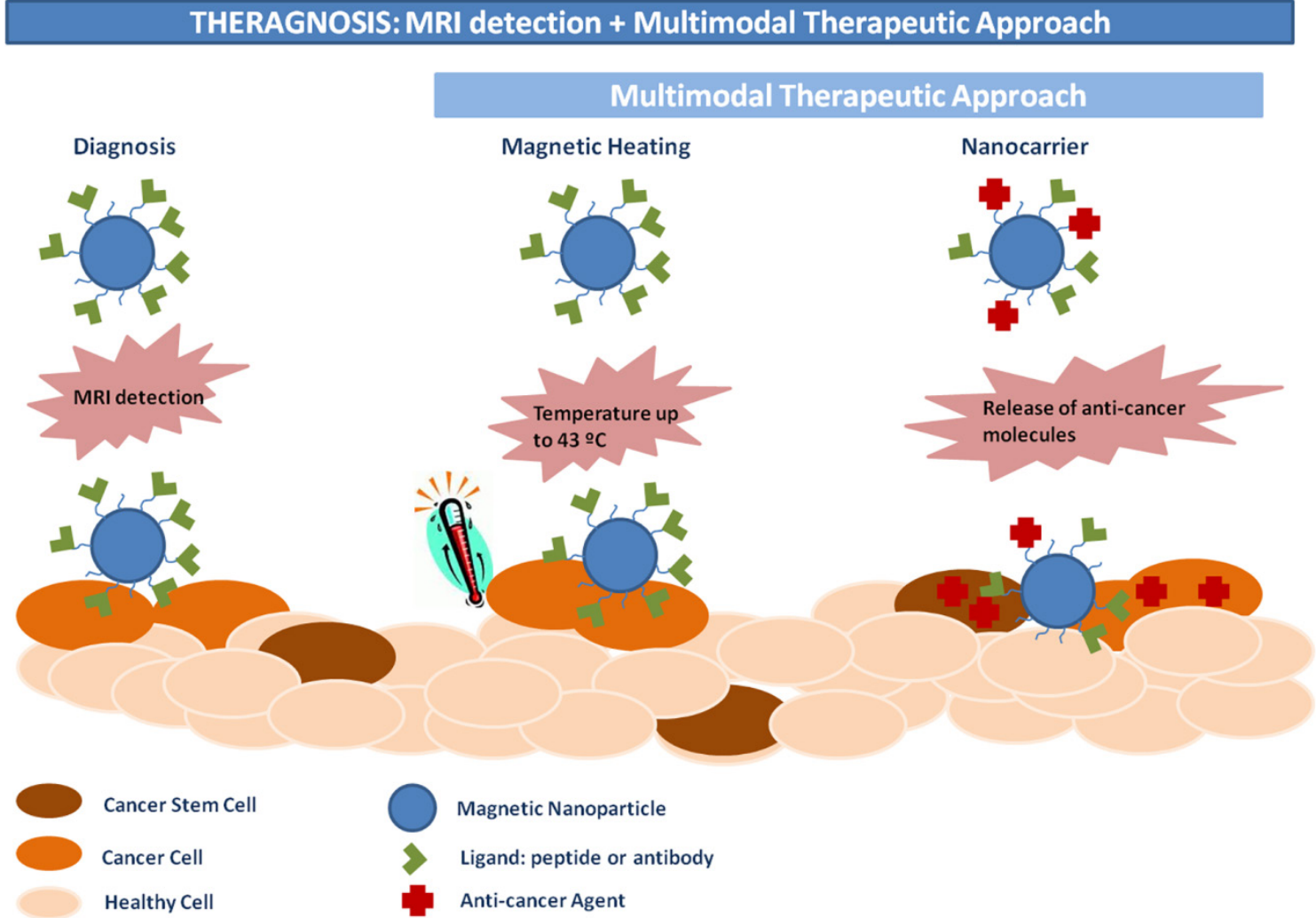

Figure 4. Example of magnetic nanoparticle for theranostic applications (http://www.multifun-project.eu/). Reproduced with permission from Drs. Terán and Somoza (IMDEA-Nanociencia, Madrid, Spain).

Table 3: A non-exhausting table of projects involving iron oxide nanoparticles or imaging techniques funded by the Seventh Framework programme under the European commission.[109]

\begin{tabular}{lll}
\hline Project & Consortium & Aim \\
\hline NanoDiara & 15 groups & SPIONs in detection of Rheumatoid Arthritis and Osteoarthritis \\
NAD & 19 groups & Treatment of Alzheimer's disease \\
Vibrant & 10 groups & Contrast agent for imaging of pancreatic beta-cells in Diabetes Mellitus type I \\
3Micron & 10 groups & Micro balloons in contrast agent and imaging for diagnosis of inflammatory or vascular diseases \\
NanoTher & 18 groups & Functionalization of nanoparticles for improving cancer diagnosis and therapy \\
Magnifyco & 11 groups & Theranostic potential of magnetic nanoparticles for theranostic applications in ovarian cancer \\
Nanomagdye & 8 groups & Iron oxide nanoparticles as new contrast agent in lymph node imaging of cancer cases \\
Namdiatream & 22 groups & Detection and imaging of molecular biomarkers \\
SaveMe & 19 groups & Nano core platforms to improve diagnosis and therapy in cancer \\
Multifun & 15 groups & Iron oxide nanoparticles with cancer stem cells in earlier detection of breast and pancreatic cancer \\
NanoFol & 13 groups & Improvement of treatment of inflammatory diseases by drug delivery to macrophages
\end{tabular}




\section{Perspective}

As reviewed in this article, a tremendous amount of time and money has been invested in the development of iron oxide nanoparticles for their clinical application. Recent breakthrough within areas of magnetic hyperthermia is shadowed by the simultaneous withdrawal of several existing contrast agents. Toxicity concern or lack of clinical benefit is the main cause of removal. As scientific work with nanoparticles has been attempted for approximately three decades and the already few accomplishments now are being second-guessed, it is natural to raise the concern that our resources allocated to medical research is being misplaced. Would the society benefit from iron oxide nanoparticles as contrast agents for MR imaging?

Although medical care continues to evolve, treatment resistant diseases develop constantly throughout our population, with cancer representing a significant proportion of these. Gadolinium based contrast agents still remain the current gold standard tool in achieving appropriate diagnostic sensitivity. However, these contrast agents are now not only associated with individual human toxicity, but is starting to display damaging effects on the environment.[111]

The main human toxicity related to gadolinium based agents is associated with patients suffering from pre-existing kidney failure. The patient is then at risk of developing a condition called Nephrogenic Systemic Fibrosis (NSF), developing a systemic tissue fibrosis.[112, 113] Although a causative effect never has been established between the condition and Gadolinium based agents, a positive correlation is observed in the literature. It is clear that most patients developing the condition are patients previously having received gadolinium based agents. Furthermore, gadolinium has also been found in several tissue biopsies of specimens with pre-diagnosed NSF.[112, 113] Screening the patients for renal function before administering the contrast agent, and excluding all patients with impaired function has caused the incidence of the condition to drop significantly, with no new cases reported with an onset within the last two years.[114]

Environmental concerns were raised as an increased level of gadolinium was observed in the environment since the start of the use of contrast agents for MR imaging. In most patients the contrast agent is excreted in the urine after a few hours, with little side effects occurring. However, as the waste is not collected in hospitals, and there is an insufficient purification of water waste by sewage plants, the product is detected in rivers and lakes in the environment.[115]
This is found anywhere these contrast agents are used in a clinical setting.[116] Furthermore, the substance is taken up by the plants, therefore being incorporated into the food chain for which the long-term consequences are unknown.

Therefore, it is clear that a different form of contrast agent is both necessary and beneficial for the society. A gadolinium free agent must be developed to provide all patients with diagnostic ability while maintaining environmental safety. Iron oxide nanoparticles can offer this, as they are natural compounds already existing in nature. With growing incidence of cancer, contrast agents are necessary in order to provide the best sensitivity for tumour detection and early treatment options. Therefore, focusing research on contrast agent development can be of substantial benefit for the society long-term. Furthermore, the theranostic potential of iron oxide nanoparticles are showing promising results for further cancer treatment, as seen in the recent approval of NanoTherm for hyperthermia. With the appropriate development, iron oxide nanoparticles can provide new and effective treatment methods for cancers with previously limited treatment options, both as a contrast agent, and by improving drug delivery.

\section{Conclusion}

Following the withdrawal of some iron oxide nanoparticle products from the development, a number of regulatory safeguards have been now introduced by regulatory agencies, to ensure safe and efficacious basic and clinical development of iron oxide nanoparticles. Importantly, several reflection papers have been published by the EMA providing guidance to the production companies of the process of approval. This regulatory framework is expected to guide researchers in developing, doctors in providing and patients in receiving the treatments based on iron oxide nanoparticles.

In this context, the 2020 nanomedicine research outlook as viewed by the European Commission, is to establish a stronger and faster transition of nanomedical R\&D from a laboratory to clinical development and approval.[117]

\section{Abbreviations}

Cd: cadmium; DNA: Deoxyribonucleic acid; EMA: European Medicines Agency; EPR effect: enhancement permeability and retention effect; EU: European Union; FDA: Drug Administration; FP7: seventh framework programme; FRI: fluorescence reflectance imaging; Gd: gadolinium; Gd-DTPA: gadolinium-diethylenetriamine penta-acetic acid; IOP: iron oxide particles; IONP: iron oxide nanoparticles; MMP-2: matrix metalloproteinase-2; MNPs: 
magnetic nanoparticles; MRI: Magnetic Resonance Imaging; MS: multiple sclerosis; N6L: cell surface nucleolin; NPs: nanoparticles NPs; NSF: Nephrogenic Systemic Fibrosis; PET: positron emission tomography; QD: quantum dots; RES: reticuloendothelial system; RNA: Ribonucleic acid; SPECT: single-photon emission computed tomography; SPION: Superparamagnetic iron oxide nanoparticles; USPIO: ultrasmall particles of iron oxide.

\section{Acknowledgments}

This work has been partially funded from the European Seventh Framework Programme (FP7/2007-2013) under the project MULTIFUN grant agreement no. 262943. Additional funding was for A. P-M. from EU FP7 project NAMDIATREAM (ref 246479).

\section{Competing Interests}

The authors have declared that no competing interest exists.

\section{References}

1. [Internet] Feynman RP. http://calteches.library.caltech.edu/1976/1/ 1960Bottom.pdf.

2. Fu A, Gu W, Larabell C, Alivisatos AP. Semiconductor nanocrystals for biological imaging. Current opinion in neurobiology. 2005; 15: 568-75. doi:10.1016/j.conb.2005.08.004.

3. Bruchez M, Moronne M, Gin P, Weiss S, Alivisatos AP Semiconductor Nanocrystals as Fluorescent Biological Labels. Science. 1998; 281: 2013-6. doi:10.1126/science.281.5385.2013.

4. Corot C, Robert P, Idee JM, Port M. Recent advances in iron oxide nanocrystal technology for medical imaging. Adv Drug Deliv Rev. 2006; 58: 1471-504. doi:10.1016/j.addr.2006.09.013.

5. Stoll G, Bendszus M. Imaging of inflammation in the peripheral and central nervous system by magnetic resonance imaging. Neuroscience. 2009; 158: 1151-60. doi:10.1016/j.neuroscience.2008.06.045.

6. Vellinga MM, Oude Engberink RD, Seewann A, Pouwels PJ, Wattjes MP, van der Pol SM, et al. Pluriformity of inflammation in multiple sclerosis shown by ultra-small iron oxide particle enhancement. Brain : a journal of neurology. 2008; 131: 800-7. doi:10.1093/brain/awn009.

7. Zhen Z, Xie J. Development of manganese-based nanoparticles as contrast probes for magnetic resonance imaging. Theranostics. 2012; 2: 45-54. doi:10.7150/thno.3448

8. Sun C, Lee JS, Zhang M. Magnetic nanoparticles in MR imaging and drug delivery. Adv Drug Deliv Rev. 2008; 60: 1252-65. doi:10.1016/j.addr.2008.03.018

9. Gutwein LG, Webster TJ. Increased viable osteoblast density in the presence of nanophase compared to conventional alumina and titania particles. Biomaterials. 2004; 25: 4175-83. doi:10.1016/j.biomaterials.2003.10.090.

10. Webster T, Ahn E. Nanostructured Biomaterials for Tissue Engineering Bone. In: Lee K, Kaplan D, editors. Tissue Engineering II. Heidelberg: Springer Berlin. 2007: 275-308

11. Chakraborty M, Jain S, Rani V. Nanotechnology: emerging tool for diagnostics and therapeutics. Applied biochemistry and biotechnology. 2011; 165: 1178-87. doi:10.1007/s12010-011-9336-6.

12. Kateb B, Chiu K, Black KL, Yamamoto V, Khalsa B, Ljubimova JY, et al. Nanoplatforms for constructing new approaches to cancer treatment, imaging, and drug delivery: what should be the policy? NeuroImage. 2011; 54 Suppl 1: S106-24. doi:10.1016/j.neuroimage.2010.01.105

13. Bhaskar S, Tian F, Stoeger T, Kreyling W, de la Fuente JM, Grazu V, et al. Multifunctional Nanocarriers for diagnostics, drug delivery and targeted treatment across blood-brain barrier: perspectives on tracking and neuroimaging. Particle and fibre toxicology. 2010; 7: 3. doi:10.1186/1743-8977-7-3.

14. Liu C, Zhang N. Nanoparticles in gene therapy principles, prospects, and challenges. Progress in molecular biology and translational science. 2011; 104: 509-62 doi:10.1016/B978-0-12-416020-0.00013-9.

15. Parveen S, Misra R, Sahoo SK. Nanoparticles: a boon to drug delivery, therapeutics, diagnostics and imaging. Nanomedicine-Uk. 2012; 8: 147-66. doi:10.1016/j.nano.2011.05.016.
16. Shenoi MM, Shah NB, Griffin RJ, Vercellotti GM, Bischof JC. Nanoparticle preconditioning for enhanced thermal therapies in cancer. Nanomedicine (Lond). 2011; 6: 545-63. doi:10.2217/nnm.10.153.

17. Shinkai M, Yanase M, Suzuki M, Hiroyuki H, Wakabayashi T, Yoshida J, et al. Intracellular hyperthermia for cancer using magnetite cationic liposomes. Journal of Magnetism and Magnetic Materials. 1999; 194: 176-84. doi:10.1016/S0304-8853(98)00586-1.

18. Kim DH, Nikles DE, Brazel CS. Synthesis and Characterization of Multifunctional Chitosan- MnFe2O4 Nanoparticles for Magnetic Hyperthermia and Drug Delivery. Materials. 2010; 3: 4051-65. doi:10.3390/ma3074051.

19. Zhao Y, Alakhova DY, Kabanov AV. Can nanomedicines kill cancer stem cells? Adv Drug Deliv Rev. 2013; 65: 1763-83. doi:10.1016/j.addr.2013.09.016.

20. Sadhukha T, Niu L, Wiedmann TS, Panyam J. Effective elimination of cancer stem cells by magnetic hyperthermia. Molecular pharmaceutics. 2013; 10: 1432-41. doi:10.1021/mp400015b.

21. Yang C, Xiong F, Wang J, Dou J, Chen J, Chen D, et al. Anti-ABCG2 monoclonal antibody in combination with paclitaxel nanoparticles against cancer stem-like cell activity in multiple myeloma. Nanomedicine (Lond). 2014; 9: 45-60. doi:10.2217/nnm.12.216.

22. Conde J, Dias JT, Grazu V, Moros M, Baptista PV, de la Fuente JM. Revisiting 30 years of biofunctionalization and surface chemistry of inorganic nanoparticles for nanomedicine. Front Chem. 2014; 2: 48. doi:10.3389/fchem.2014.00048.

23. Kievit FM, Zhang M. Surface engineering of iron oxide nanoparticles for targeted cancer therapy. Acc Chem Res. 2011; 44: 853-62. doi:10.1021/ar2000277.

24. Yigit MV, Moore A, Medarova Z. Magnetic nanoparticles for cancer diagnosis and therapy. Pharmaceutical research. 2012; 29: 1180-8. doi:10.1007/s11095-012-0679-7.

25. Movia D, Poland C, Tran L, Volkov Y, Prina-Mello A. Multilayered Nanoparticles for Personalized Medicine: Translation into Clinical Markets. In: Handbook of Clinical Nanomedicine: Nanoparticles, Imaging, Therapy and Clinical Applications Singapore: Pan Stanford Publishing Pte Ltd; 2016.

26. Kumar A, Jena PK, Behera S, Lockey RF, Mohapatra S, Mohapatra S. Multifunctional magnetic nanoparticles for targeted delivery. Nanomedicine-Uk. 2010; 6: 64-9. doi:10.1016/j.nano.2009.04.002

27. Salas G, Casado C, Teran FJ, Miranda R, Serna CJ, Morales MP. Controlled synthesis of uniform magnetite nanocrystals with high-quality properties for biomedical applications. J Mater Chem. 2012; 22: 21065-75. doi:10.1039/C2JM34402E.

28. Aitziber L. Cortajarena DO, Sandra M. Ocampo, Alberto Gonzalez-García, Pierre Couleaud, Rodolfo Miranda, Cristobal Belda-Iniesta and Angel Ayuso-Sacido. Engineering Iron Oxide Nanoparticles for Clinical Settings. Nanobiomedicine. 2014; 1:2. doi:doi: 10.5772/58841.

29. Ruiz A, Morais P, Bentes de Azevedo R, Lacava ZM, Villanueva A, del Puerto Morales M. Magnetic nanoparticles coated with dimercaptosuccinic acid: development, characterization, and application in biomedicine. J Nanopart Res. 2014; 16: 1-20. doi:10.1007/s11051-014-2589-6.

30. Marciello M, Connord V, Veintemillas-Verdaguer S, Verges MA, Carrey J, Respaud M, et al. Large scale production of biocompatible magnetite nanocrystals with high saturation magnetization values through green aqueous synthesis. Journal of Materials Chemistry B. 2013; 1: 5995-6004. doi:10.1039/С3ТВ20949K.

31. Gobbo OL, Wetterling F, Vaes P, Teughels S, Markos F, Edge D, et al. Biodistribution and pharmacokinetic studies of SPION using particle electron paramagnetic resonance, MRI and ICP-MS. Nanomedicine-Uk. 2015; 10: 1751-60. doi:10.2217/nnm.15.22.

32. Hilger I, Kaiser WA. Iron oxide-based nanostructures for MRI and magnetic hyperthermia. Nanomedicine-Uk. 2012; 7: 1443-59. doi:10.2217/nnm.12.112.

33. Markides H, Rotherham M, El Haj AJ. Biocompatibility and Toxicity of Magnetic Nanoparticles in Regenerative Medicine. J Nanomater. 2012; 2012: 11. doi:10.1155/2012/614094.

34. Calero M, Gutiérrez L, Salas G, Luengo $\mathrm{Y}$, Lázaro A, Acedo P, et al. Efficient and safe internalization of magnetic iron oxide nanoparticles: Two fundamental requirements for biomedical applications. Nanomedicine: Nanotechnology, Biology and Medicine. 2014; 10: 733-43. doi:http://dx.doi.org/10.1016/j.nano.2013.11.010.

35. Ruiz A, Hernandez Y, Cabal C, Gonzalez E, Veintemillas-Verdaguer S, Martinez $\mathrm{E}$, et al. Biodistribution and pharmacokinetics of uniform magnetite nanoparticles chemically modified with polyethylene glycol. Nanoscale. 2013; 5: 11400-8. doi:10.1039/C3NR01412F.

36. Latorre A, Couleaud P, Aires A, Cortajarena AL, Somoza Á. Multifunctionalization of magnetic nanoparticles for controlled drug release: A general approach. European Journal of Medicinal Chemistry. 2014; 82: 355-62. doi:10.1016/j.ejmech.2014.05.078.

37. Mulens V, Morales MdP, Barber DF. Development of Magnetic Nanoparticles for Cancer Gene Therapy: A Comprehensive Review. ISRN Nanomaterials. 2013; 1: 1-14. doi:10.1155/2013/646284.

38. Arruebo M, Valladares M, Gonzalez-Fernandez A. Antibody-Conjugated Nanoparticles for Biomedical Applications. J Nanomater. 2009; 1: 1-24. doi:10.1155/2009/439389.

39. Destouches D, El Khoury D, Hamma-Kourbali Y, Krust B, Albanese P, Katsoris $\mathrm{P}$, et al. Suppression of Tumor Growth and Angiogenesis by a Specific Antagonist of the Cell-Surface Expressed Nucleolin. PloS one. 2008; 3: e2518. doi:10.1371/journal.pone.0002518 
40. Ventola CL. The Nanomedicine Revolution: Part 1: Emerging Concepts. Pharmacy and Therapeutics. 2012; 37: 512-25.

41. [Internet] WHO/IARC: Lyon, France. World Cancer Report 2008. http://www.iarc.fr/en/publications/pdfs-online/wcr/2008/wcr_2008.pdf.

42. Weissleder R. Molecular imaging in cancer. Science. 2006; 312: 1168-71. doi:10.1126/science.1125949.

43. Condeelis J, Weissleder R. In vivo imaging in cancer. Cold Spring Harbor perspectives in biology. 2010; 2: 1-24. doi:10.1101/cshperspect.a003848.

44. Ambrosone A, Mattera L, Marchesano V, Quarta A, Susha AS, Tino A, et al. Mechanisms underlying toxicity induced by CdTe quantum dots determined in an invertebrate model organism. Biomaterials. 2012; 33: 1991-2000. doi:10.1016/j.biomaterials.2011.11.041.

45. Gobbo OL, Zurek M, Tewes F, Ehrhardt C, Cremillieux Y. Manganese: a new contrast agent for lung imaging? Contrast media \& molecular imaging. 2012; 7: 542-6. doi:10.1002/cmmi.1483.

46. Runge VM, Ai T, Hao D, Hu X. The developmental history of the gadolinium chelates as intravenous contrast media for magnetic resonance. Investigative radiology. 2011; 46: 807-16. doi:10.1097/RLI.0b013e318237913b.

47. Aime S, Reineri F. Advances in bio-imaging: a survey from WWMR 2010. Journal of magnetic resonance. 2011; 209: 109-15. doi:10.1016/j.jmr.2011.02.021.

48. Mosbah K, Ruiz-Cabello J, Berthezene Y, Cremillieux Y. Aerosols and gaseous contrast agents for magnetic resonance imaging of the lung. Contrast media \& molecular imaging. 2008; 3: 173-90. doi:10.1002/cmmi.252.

49. Rumenapp C, Gleich B, Haase A. Magnetic nanoparticles in magnetic resonance imaging and diagnostics. Pharmaceutical research. 2012; 29: 1165-79. doi:10.1007/s11095-012-0711-y

50. Na HB, Song IC, Hyeon T. Inorganic Nanoparticles for MRI Contrast Agents. Advanced Materials. 2009; 21: 2133-48. doi:10.1002/adma.200802366.

51. Yallapu MM, Foy SP, Jain TK, Labhasetwar V. PEG-functionalized magnetic nanoparticles for drug delivery and magnetic resonance imaging applications. Pharmaceutical research. 2010; 27: 2283-95. doi:10.1007/s11095-010-0260-1.

52. Hayashi K, Nakamura M, Sakamoto W, Yogo T, Miki H, Ozaki S, et al. Superparamagnetic nanoparticle clusters for cancer theranostics combining magnetic resonance imaging and hyperthermia treatment. Theranostics. 2013; 3: 366-76. doi: $10.7150 /$ thno. 5860.

53. Zhao Q, Wang L, Cheng R, Mao L, Arnold RD, Howerth EW, et al. Magnetic nanoparticle-based hyperthermia for head \& neck cancer in mouse models. Theranostics. 2012; 2: 113-21. doi:10.7150/thno.3854.

54. Peer D, Karp JM, Hong S, Farokhzad OC, Margalit R, Langer R. Nanocarriers as an emerging platform for cancer therapy. Nature nanotechnology. 2007; 2: 751-60. doi:10.1038/nnano.2007.387.

55. Krown SE, Northfelt DW, Osoba D, Stewart JS. Use of liposomal anthracyclines in Kaposi's sarcoma. Seminars in oncology. 2004; 31: 36-52. doi:10.1053/j.seminoncol.2004.08.003.

56. Ranganathan R, Madanmohan S, Kesavan A, Baskar G, Krishnamoorthy YR, Santosham R, et al. Nanomedicine: towards development of patient-friendly drug-delivery systems for oncological applications. International journal of nanomedicine. 2012; 7: 1043-60. doi:10.2147/IJN.S25182.

57. Gaspar MM, Radomska A, Gobbo OL, Bakowsky U, Radomski MW, Ehrhardt C. Targeted delivery of transferrin-conjugated liposomes to an orthotopic model of lung cancer in nude rats. Journal of aerosol medicine and pulmonary drug delivery. 2012; 25: 310-8. doi:10.1089/jamp.2011.0928.

58. Furlani EP. Magnetic Biotransport: Analysis and Applications. Materials. 2010; 3: 2412-46. doi:10.3390/ma3042412.

59. Schlorf T, Meincke M, Kossel E, Gluer CC, Jansen O, Mentlein R. Biological properties of iron oxide nanoparticles for cellular and molecular magnetic resonance imaging. International journal of molecular sciences. 2010; 12: 12-23. doi:10.3390/ijms12010012.

60. Al Faraj A, Lacroix G, Alsaid H, Elgrabi D, Stupar V, Robidel F, et al. Longitudinal $3 \mathrm{He}$ and proton imaging of magnetite biodistribution in a rat model of instilled nanoparticles. Magnetic resonance in medicine : official journal of the Society of Magnetic Resonance in Medicine / Society of Magnetic Resonance in Medicine. 2008; 59: 1298-303. doi:10.1002/mrm.21571.

61. Al Faraj A, Bessaad A, Cieslar K, Lacroix G, Canet-Soulas E, Cremillieux Y. Long-term follow-up of lung biodistribution and effect of instilled SWCNTs using multiscale imaging techniques. Nanotechnology. 2010; 21: 175103. doi:10.1088/0957-4484/21/17/175103

62. Barraud L, Merle P, Soma E, Lefrancois L, Guerret S, Chevallier M, et al. Increase of doxorubicin sensitivity by doxorubicin-loading into nanoparticles for hepatocellular carcinoma cells in vitro and in vivo. Journal of hepatology. 2005; 42: 736-43. doi:10.1016/j.jhep.2004.12.035.

63. Han HD, Mangala LS, Lee JW, Shahzad MM, Kim HS, Shen D, et al. Targeted gene silencing using RGD-labeled chitosan nanoparticles. Clinical cancer research : an official journal of the American Association for Cancer Research. 2010; 16: 3910-22. doi:10.1158/1078-0432.CCR-10-0005.

64. Parveen S, Mitra M, Krishnakumar S, Sahoo SK. Enhanced antiproliferative activity of carboplatin-loaded chitosan-alginate nanoparticles in a retinoblastoma cell line. Acta biomaterialia. 2010; 6: 3120-31. doi:10.1016/j.actbio.2010.02.010

65. Maeng JH, Lee DH, Jung KH, Bae YH, Park IS, Jeong S, et al. Multifunctional doxorubicin loaded superparamagnetic iron oxide nanoparticles for chemotherapy and magnetic resonance imaging in liver cancer. Biomaterials. 2010; 31: 4995-5006. doi:10.1016/j.biomaterials.2010.02.068.
66. Schwerdt JI, Goya GF, Calatayud MP, Herenu CB, Reggiani PC, Goya RG. Magnetic field-assisted gene delivery: achievements and therapeutic potential. Current gene therapy. 2012; 12: 116-26. doi:10.2174/156652312800099616.

67. Li C, Li L, Keates AC. Targeting cancer gene therapy with magnetic nanoparticles. Oncotarget. 2012; 3: 365-70.

68. Yan JF, Liu J. Nanocryosurgery and its mechanisms for enhancing freezing efficiency of tumor tissues. Nanomedicine-Uk. 2008; 4: 79-87. doi:10.1016/j.nano.2007.11.002

69. Isbert C, Ritz JP, Roggan A, Schuppan D, Ruhl M, Buhr HJ, et al. Enhancement of the immune response to residual intrahepatic tumor tissue by laser-induced thermotherapy (LITT) compared to hepatic resection. Lasers in surgery and medicine. 2004; 35: 284-92. doi:10.1002/lsm.20097.

70. Ratto F, Matteini P, Centi S, Rossi F, Pini R. Gold nanorods as new nanochromophores for photothermal therapies. Journal of biophotonics. 2011; 4: 64-73. doi:10.1002/jbio.201000002.

71. Kennedy JE, Wu F, ter Haar GR, Gleeson FV, Phillips RR, Middleton MR, et al. High-intensity focused ultrasound for the treatment of liver tumours. Ultrasonics. 2004; 42: 931-5. doi:10.1016/j.ultras.2004.01.089.

72. Kim J, Chung DJ, Jung SE, Cho SH, Hahn ST, Lee JM. Therapeutic effect of high-intensity focused ultrasound combined with transarterial chemoembolisation for hepatocellular carcinoma $<5 \mathrm{~cm}$ : comparison with transarterial chemoembolisation monotherapy--preliminary observations. The British journal of radiology. 2012; 85: 940-6. doi:10.1259/bjr/32750755.

73. Chen MH, Yang W, Yan K, Zou MW, Solbiati L, Liu JB, et al. Large liver tumors: protocol for radiofrequency ablation and its clinical application in 110 patients--mathematic model, overlapping mode, and electrode placement process. Radiology. 2004; 232: 260-71. doi:10.1148/radiol.2321030821.

74. Fu Y, Yang W, Wu W, Yan K, Xing BC, Chen MH. Radiofrequency ablation in the management of unresectable intrahepatic cholangiocarcinoma. Journal of vascular and interventional radiology : JVIR. 2012; 23: 642-9. doi:10.1016/j.jvir.2012.01.081.

75. Hilger I, Hergt R, Kaiser WA. Use of magnetic nanoparticle heating in the treatment of breast cancer. IEE proceedings Nanobiotechnology. 2005; 152: 33-9. doi:10.1049/ip-nbt:20055018.

76. Kettering M, Richter H, Wiekhorst F, Bremer-Streck S, Trahms L, Kaiser WA, et al. Minimal-invasive magnetic heating of tumors does not alter intra-tumoral nanoparticle accumulation, allowing for repeated therapy sessions: an in vivo study in mice. Nanotechnology. 2011; 22: 505102. doi:10.1088/0957-4484/22/50/505102

77. Huang HS, Hainfeld JF. Intravenous magnetic nanoparticle cancer hyperthermia. International journal of nanomedicine. 2013; 8: 2521-32. doi:10.2147/IJN.S43770.

78. Day ES, Morton JG, West JL. Nanoparticles for thermal cancer therapy. Journal of biomechanical engineering. 2009; 131: 074001. doi:10.1115/1.3156800.

79. Huang N, Wang H, Zhao J, Lui H, Korbelik M, Zeng H. Single-wall carbon nanotubes assisted photothermal cancer therapy: animal study with a murine model of squamous cell carcinoma. Lasers in surgery and medicine. 2010; 42: 638-48. doi:10.1002/lsm.20968.

80. Li Y, Dong H, Li Y, Shi D. Graphene-based nanovehicles for photodynamic medical therapy. International journal of nanomedicine. 2015; 10: 2451-9. doi:10.2147/ijn.s68600.

81. [Internet] www.magforce.de/fileadmin/magforce/5_presse/presentations/Company_ presentation_May2013_English.pdf.

82. Maier-Hauff $K$, Ulrich $F$, Nestler $D$, Niehoff $H$, Wust $P$, Thiesen $B$, et al. Efficacy and safety of intratumoral thermotherapy using magnetic iron-oxide nanoparticles combined with external beam radiotherapy on patients with recurrent glioblastoma multiforme. Journal of neuro-oncology. 2011; 103: 317-24. doi:10.1007/s11060-010-0389-0.

83. Jordan A, Scholz R, Maier-Hauff K, van Landeghem FK, Waldoefner N, Teichgraeber $\mathrm{U}$, et al. The effect of thermotherapy using magnetic nanoparticles on rat malignant glioma. Journal of neuro-oncology. 2006; 78: 7-14. doi:10.1007/s11060-005-9059-z.

84. Johannsen M, Gneveckow U, Taymoorian K, Thiesen B, Waldofner N, Scholz $\mathrm{R}$, et al. Morbidity and quality of life during thermotherapy using magnetic nanoparticles in locally recurrent prostate cancer: results of a prospective phase I trial. International journal of hyperthermia : the official journal of European Society for Hyperthermic Oncology, North American Hyperthermia Group 2007; 23: 315-23 doi:10.1080/02656730601175479.

85. Veiseh O, Gunn JW, Zhang M. Design and fabrication of magnetic nanoparticles for targeted drug delivery and imaging. Adv Drug Deliv Rev. 2010; 62: 284-304. doi:10.1016/j.addr.2009.11.002.

86. $\mathrm{He} \mathrm{C,} \mathrm{Hu} \mathrm{Y,} \mathrm{Yin} \mathrm{L,} \mathrm{Tang} \mathrm{C,} \mathrm{Yin} \mathrm{C.} \mathrm{Effects} \mathrm{of} \mathrm{particle} \mathrm{size} \mathrm{and} \mathrm{surface} \mathrm{charge} \mathrm{on}$ cellular uptake and biodistribution of polymeric nanoparticles. Biomaterials. 2010; 31: 3657-66. doi:10.1016/j.biomaterials.2010.01.065.

87. Alexiou C, Jurgons R, Seliger C, Brunke O, Iro H, Odenbach S. Delivery of superparamagnetic nanoparticles for local chemotherapy after intraarterial infusion and magnetic drug targeting. Anticancer research. 2007; 27: 2019-22.

88. Tietze R, Lyer S, Durr S, Alexiou C. Nanoparticles for cancer therapy using magnetic forces. Nanomedicine (Lond). 2012; 7: 447-57. doi: $10.2217 / \mathrm{nnm} .12 .10$

89. Lubbe AS, Alexiou C, Bergemann C. Clinical applications of magnetic drug targeting. The Journal of surgical research. 2001; 95: 200-6. doi:10.1006/jsre. 2000.6030 
90. Dames P, Gleich B, Flemmer A, Hajek K, Seidl N, Wiekhorst F, et al. Targeted delivery of magnetic aerosol droplets to the lung. Nature nanotechnology. 2007; 2: 495-9. doi:10.1038/nnano.2007.217.

91. Fay F, Scott CJ. Antibody-targeted nanoparticles for cancer therapy. Immunotherapy. 2011; 3: 381-94. doi:10.2217/imt.11.5.

92. Arosio D, Casagrande C, Manzoni L. Integrin-mediated drug delivery in cancer and cardiovascular diseases with peptide-functionalized nanoparticles. Current medicinal chemistry. 2012; 19: 3128-51. doi:10.2174/092986712800784748.

93. Huang R, Han L, Li J, Liu S, Shao K, Kuang Y, et al. Chlorotoxin-modified macromolecular contrast agent for MRI tumor diagnosis. Biomaterials. 2011; 32: 5177-86. doi:10.1016/j.biomaterials.2011.03.075.

94. Destouches D, Page N, Hamma-Kourbali Y, Machi V, Chaloin O, Frechault S, et al. A simple approach to cancer therapy afforded by multivalent pseudopeptides that target cell-surface nucleoproteins. Cancer research. 2011; 71: 3296-305. doi:10.1158/0008-5472.CAN-10-3459.

95. Manke A, Wang L, Rojanasakul Y. Mechanisms of nanoparticle-induced oxidative stress and toxicity. BioMed research international. 2013; 1: 942916. doi:10.1155/2013/942916.

96. Mahmoudi M, Hofmann H, Rothen-Rutishauser B, Petri-Fink A. Assessing the in vitro and in vivo toxicity of superparamagnetic iron oxide nanoparticles. Chemical reviews. 2012; 112: 2323-38. doi:10.1021/cr2002596.

97. Ehmann F, Sakai-Kato K, Duncan R, Hernan Perez de la Ossa D, Pita R, Vidal $\mathrm{JM}$, et al. Next-generation nanomedicines and nanosimilars: EU regulators' initiatives relating to the development and evaluation of nanomedicines. Nanomedicine (Lond). 2013; 8: 849-56. doi:10.2217/nnm.13.68.

98. [Internet] European Medicines Agency. Regulatory and procedural guideline. 2009. http://www.ema.europa.eu/docs/en_GB/document_library/ Regulatory_and_procedural_guideline/2009/10/WC500004481.pdf.

99. [Internet] EU legislation on medical devices: Guidance document. 2012. http://ec.europa.eu/health/medical-devices/files/meddev/2_1_6_ol_en.pdf

100. [Internet] AFSSAPS: Recommandations for toxicological evaluation of nanoparticle medicinal products. 2011. http://ansm.sante.fr/var/ansm_site/ storage/original/application/2968a90b774b563b03405379b7d4f4e6.pdf.

101. Mahmoudi M, Simchi A, Imani M, Shokrgozar MA, Milani AS, Hafeli UO, et al. A new approach for the in vitro identification of the cytotoxicity of superparamagnetic iron oxide nanoparticles. Colloids and surfaces B, Biointerfaces. 2010; 75: 300-9. doi:10.1016/j.colsurfb.2009.08.044.

102. [Internet] European Medicines Agency: Reflection paper on non-clinical studies for generic nanoparticle iron medicinal product applications. 2011. http://www.ema.europa.eu/docs/en_GB/document_library/Scientific_guid eline/2011/04/WC500105048.pdf.

103. [Internet] European Medicines Agency: Reflection paper on surface coatings: general issues for consideration regarding parenteral administration of coated nanomedicine products. 2013. http://www.ema.europa.eu/docs/ en_GB/document_library/Scientific_guideline/2013/08/WC500147874.pdf.

104. [Internet] European Medicines Agency: Guideline on clinical evaluation of diagnostic agents. 2009. http://www.ema.europa.eu/docs/en_GB/ document_library/Scientific_guideline/2009/09/WC500003580.pdf.

105. Ahmed N, Fessi H, Elaissari A. Theranostic applications of nanoparticles in cancer. Drug discovery today. 2012; 17: 928-34. doi:10.1016/j.drudis.2012.03.010.

106. Liu Y, Zhang N. Gadolinium loaded nanoparticles in theranostic magnetic resonance imaging. Biomaterials. 2012; 33: 5363-75. doi:10.1016/j.biomaterials.2012.03.084

107. Xu H, Cheng L, Wang C, Ma X, Li Y, Liu Z. Polymer encapsulated upconversion nanoparticle/iron oxide nanocomposites for multimodal imaging and magnetic targeted drug delivery. Biomaterials. 2011; 32: 9364-73. doi:10.1016/j.biomaterials.2011.08.053

108. Hayashi K, Nakamura M, Miki H, Ozaki S, Abe M, Matsumoto T, et al. Magnetically responsive smart nanoparticles for cancer treatment with a combination of magnetic hyperthermia and remote-control drug release. Theranostics. 2014; 4: 834-44. doi:10.7150/thno.9199.

109. [Internet] European Technological Platform on Nanomedicine: European Funding allocated to Nanomedicine-related projects. 2015. http://www.etp-nanomedicine.eu/public/about-nanomedicine/european-f unding.

110. [Internet] The European Technology Platform on Nanomedicine. 2014. http:// www.etp-nanomedicine.eu/public.

111. Nofiele JT, Haedicke IE, Zhu Yle K, Zhang XA, Cheng HL. Gadolinium-free extracellular MR contrast agent for tumor imaging. Journal of magnetic resonance imaging : JMRI. 2015; 41: 397-403. doi:10.1002/jmri.24561.

112. Kuo PH, Kanal E, Abu-Alfa AK, Cowper SE. Gadolinium-based MR contrast agents and nephrogenic systemic fibrosis. Radiology. 2007; 242: 647-9. doi:10.1148/radiol.2423061640.

113. Longmire M, Choyke PL, Kobayashi H. Clearance properties of nano-sized particles and molecules as imaging agents: considerations and caveats. Nanomedicine (Lond). 2008; 3: 703-17. doi:10.2217/17435889.3.5.703.

114. Prince MR, Zhang HL, Roditi GH, Leiner T, Kucharczyk W. Risk factors for NSF: a literature review. Journal of magnetic resonance imaging : JMRI. 2009; 30: 1298-308. doi:10.1002/jmri.21973.

115. Bau M, Dulski P. Anthropogenic origin of positive gadolinium anomalies in river waters. Earth and Planetary Science Letters. 1996; 143: 245-55. doi:10.1016/0012-821X(96)00127-6.
116. Elbaz-Poulichet F, Seidel JL, Othoniel C. Occurrence of an anthropogenic gadolinium anomaly in river and coastal waters of southern France. Water research. 2002; 36: 1102-5. doi:10.1016/S0043-1354(01)00370-0.

117. [Internet] European Technological Platform on Nanomedicine: Nanomedicine 2020. White paper to the horizon 2020 Framework Programme for Research and Innovation. 2013. http://www.etp-nanomedicine.eu/public/ press-documents/publications/etpn-publications/etpn-white-paper-H2020. 